\title{
A SOLAR AIR COLLECTOR WITH INTEGRATED LATENT HEAT THERMAL STORAGE
}

\begin{abstract}
Pavel CHARVAT ${ }^{1}$, Milan OSTRY ${ }^{2}$, Tomas MAUDER ${ }^{3}$, Lubomir KLIMES ${ }^{4}$
Abstract: Simulations of the behaviour of a solar air collector with integrated latent heat thermal storage were performed. The model of the collector was created with the use of coupling between TRNSYS 17 and MATLAB. Latent heat storage (Phase Change Material - PCM) was integrated with the solar absorber. The model of the latent heat storage absorber was created in MATLAB and the model of the solar air collector itself was created in TRNSYS with the use of TYPE 56. The model of the latent heat storage absorber allows specification of the PCM properties as well as other parameters. The simulated air collector was the front and back pass collector with the absorber in the middle of the air cavity. Two variants were considered for comparison; the light-weight absorber made of sheet metal and the heat-storage absorber with the PCM. Simulations were performed for the climatic conditions of the Czech Republic (using TMY weather data).
\end{abstract}

\section{INTRODUCTION}

Solar air systems - the systems where air is used as a heat carrier - are not as common as the water-based solar systems. Among the reasons there is the smaller thermal capacity of air in comparison to that of water. Nevertheless, the air solar systems can be used in many applications including space heating of buildings.

A general problem of solar thermal systems is the need for thermal storage in order to balance the supply and demand of heat over a certain period of time [1]. Water can easily be used as a heat storage medium in the water-based solar systems but air is a quite impractical heat storage medium in the case of the air-based solar systems. Therefore, some other ways of heat storage must be used with solar air-systems. Building structures can be used as a thermal storage mass but this arrangement is not suitable for all situations. The Tromb wall [2] is one example of purpose-provided thermal storage in buildings. Another possibility is the use of rock bed thermal storage where solid materials (e.g. gravel) are used to store heat [3]. The main disadvantages of rock bed storage are a rather high volume of rock beds, problematic cleaning and the pressure drop and its distribution across the storage bed. A very promising medium for thermal storage are Phase Change Materials (PCMs) [4], [5]. The PCMs are materials with high heat of fusion. That provides a very high thermal storage capacity (and also an energy storage density) in a narrow temperature interval around the melting point. Various kinds of the PCM-air heat exchangers were designed and tested (e.g. [6]).

\footnotetext{
${ }^{1}$ Pavel Charvat, Faculty of Mechanical Engineering,Technicka 2, 61669 Brno, charvat@fme.vutbr.cz

${ }^{2}$ Milan Ostry, Faculty of Civil Engineering, Veveri 95, 60200 Brno, ostry.m@fce.vutbr.cz

${ }^{3}$ Tomas Mauder, Faculty of Mech. Engineering, Technicka 2, 61669 Brno, charvat@fme.vutbr.cz

${ }^{4}$ Lubomir Klimes, Faculty of Mech. Engineering, Technicka 2, 61669 Brno, charvat@fme.vutbr.cz
} 


\section{SOLAR AIR COLLECTORS}

A solar air collector is a basic part of any active solar air system. There are many possible configurations of solar air collectors. One way of classification of air solar collectors is according to the airflow path around the solar absorber. The four basic configurations are: a front-pass collector, a back-pass collector, a front and back pass collector and a through-pass collector. Each configuration is suitable for a different application. The back-pass collectors are usually unglazed. These types of collectors are very often used as facade elements that also reduce the transmission heat loss of a building. A glazed double facade is an example of the front-pass solar air collector. The front and back pass configuration increases the heat exchange area of a collector since the air flows on both sides of the absorber. The highest ratio between the heat exchange surface and the insolated area of the absorber can be achieved in the through-pass configuration where air flows through the cavities (channels) inside the absorber. The configurations of the air solar collectors are shown in Figure 1.

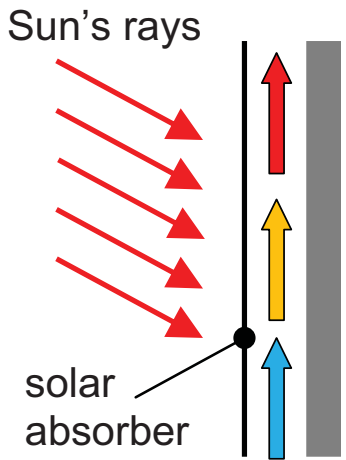

back-pass

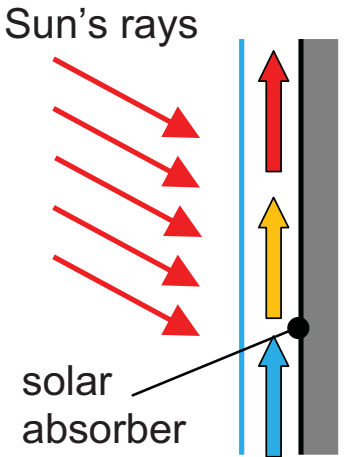

front-pass

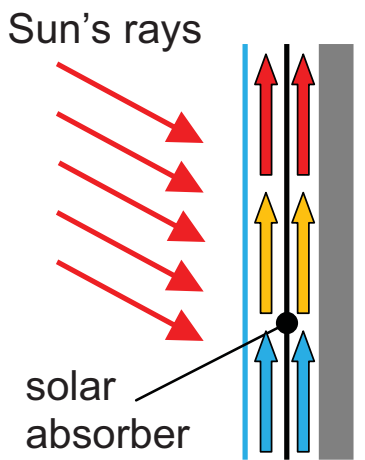

front-and-back-pass

\section{Figure: 1: Air collector configurations}

There are many possible configurations of solar air collectors with thermal storage units [7]. The front and back pass configuration was chosen for the numerical analyses because it offers a rather high heat exchange area between the solar absorber and the passing air and it is easier to be simulated than the through pass configuration. The performance of two variants of the collector was investigated. The solar absorber in the first variant was made of sheet metal and in the second variant the absorber contained a heat storage layer made of the PCM.

\section{MODEL DESCRIPTION}

TRNSYS vs. 17 was used as a simulation tool. The air collector model was created with the use of the TRNSYS TYPE 56. A model for heat transfer in the PCM heat storage layer was created in MATLAB. The transparent cover of the collector was considered to be a single pane glass. The double-glass generally increases the optical loss of the glazed part of the collector and thus it reduces the amount of solar energy reaching the absorber. On the other hand, double-glass would reduce the thermal loss of the collector. Further 
analysis is needed to decide, if or under which conditions, the double-glass would increase the overall efficiency of the air collector. The air cavities in the solar collectors were divided into 5 separate zones. Figure 2 shows the model of the collector with the individual air-zones.

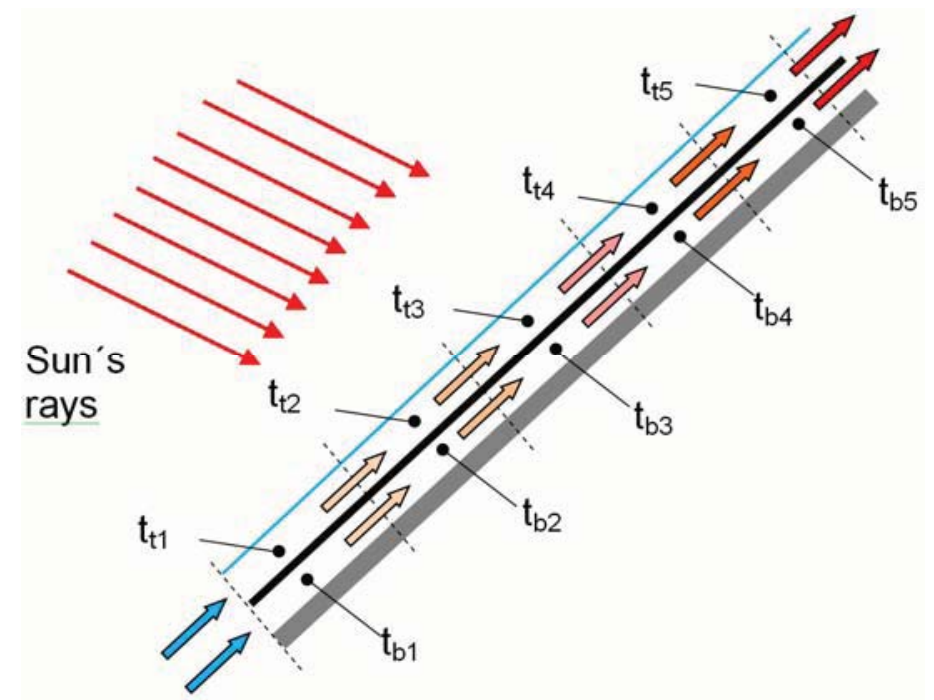

Figure 2: Model of the front and back pass collector

As already mentioned two solar absorbers were considered for a comparison. In the first case the absorber was assumed to be made of $0.5 \mathrm{~mm}$ thick sheet metal with the thermal conductivity of $40 \mathrm{~W} / \mathrm{m} \cdot \mathrm{K}$ and in the second case the absorber was assumed to consist of an aluminium container filled with the PCM with the melting temperature of $40^{\circ} \mathrm{C}$ and the latent heat of $180 \mathrm{~kJ} / \mathrm{kg}$. The enthalpy method was used to solve the phase-change of the PCM inside the absorber. Figure 3 shows the temperature-enthalpy curve of the PCM used in the simulations. No hysteresis was considered for melting and solidification.

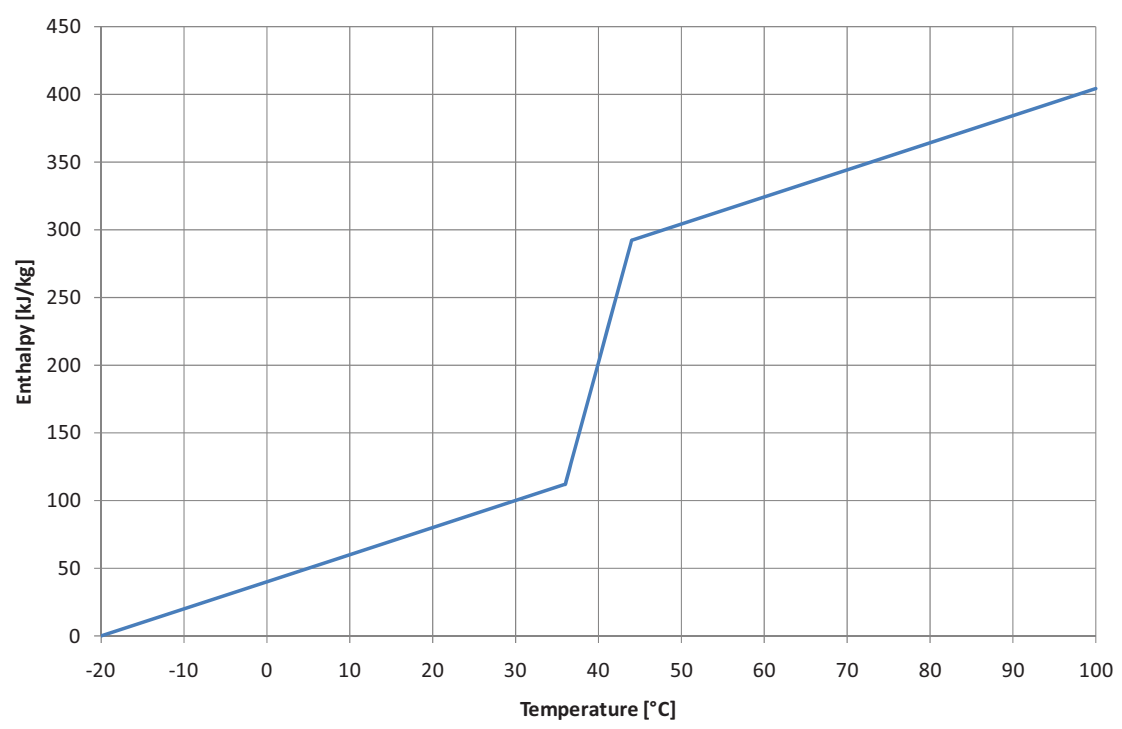

Figure 3: Enthalpy of the PCM 


\section{Results}

Weather data of the typical meteorological year (TMY) for the meteorological station Kucharovice, Czech Republic, were used in the simulation. The collectors were positioned vertically, facing due south. Figure 4 shows air temperatures at the outlet of the frontand-back pass collector with the light-weight absorber during 5 days in the spring (April 10 to April 14). As can be seen the difference between the air temperature at the outlet of insolated air cavity ( $t_{t 5}$ in Figure 2 ) is almost the same as the temperature at the outlet of the non-insulated cavity ( $t_{55}$ in Figure 2 ). This is due to the relatively small thermal resistance of the absorber (metal sheet).

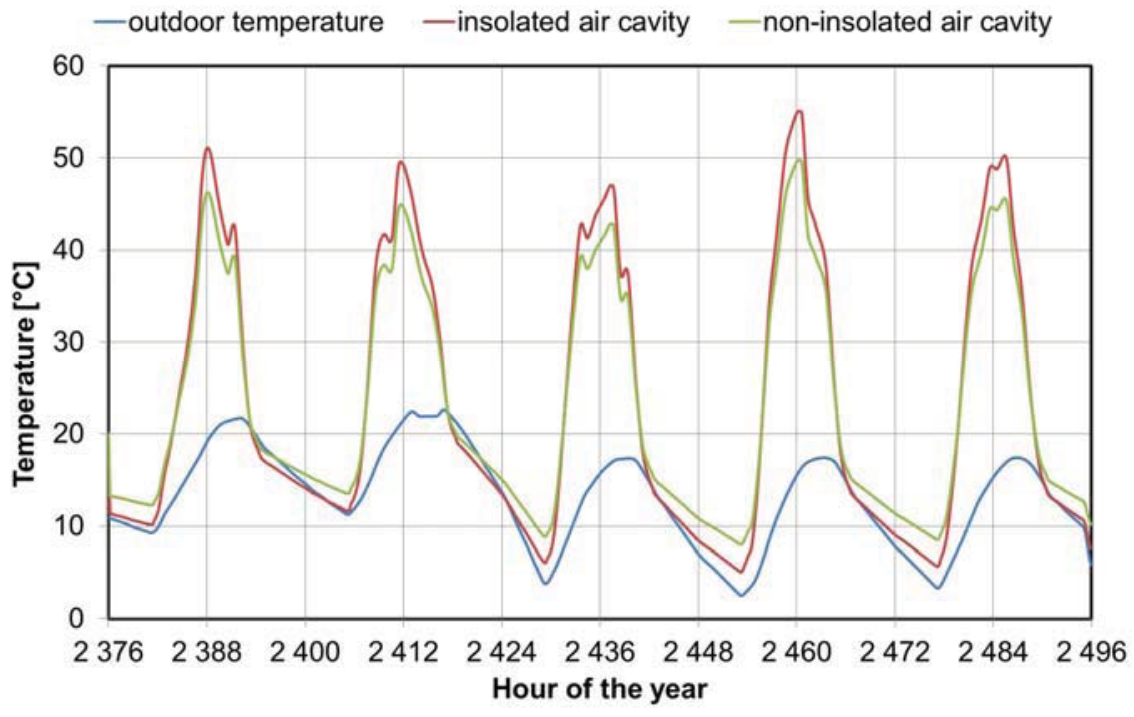

Figure 4: Air collector with the light-weight solar absorber

The air temperatures at the outlet of the collector with the absorber containing the PCM can be seen in Figure 5. The air temperatures in two air cavities differ quite significantly due to heat storage in the PCM.

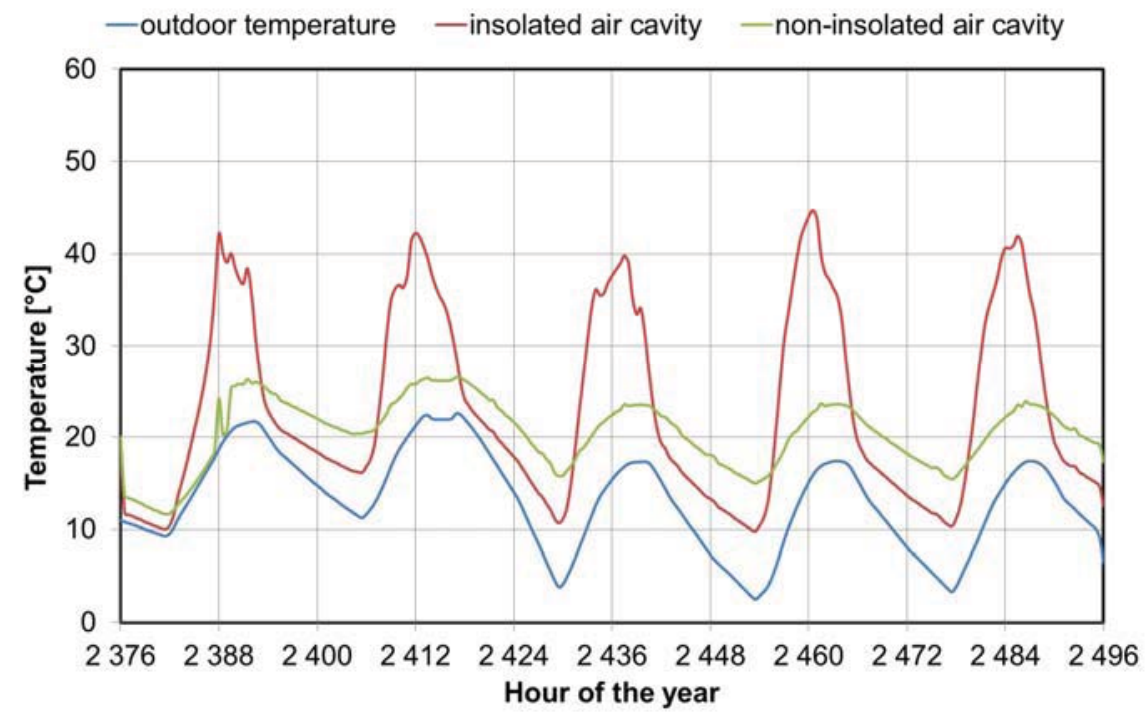

Figure 5: Air collector with the solar absorber containing the PCM 


\section{Conclusions}

The simulations of two variants of the front and back pass solar air collector were carried out. The first variant was a collector with the light-weight solar absorber made of a simple metal sheet and the other variant was a collector with the absorber containing the phase change material. As expected, thermal storage in the form of the PCM led to lower air temperatures at the outlet of the collector during the day (during sunshine hours) and higher air temperatures after the sunset when the PCM was releasing the heat stored during the day. The simulations were carried out for the PCM with the melting temperature $40^{\circ} \mathrm{C}$. More simulations are needed in order to investigate the influence of the melting temperature of the PCM, its thermal conductivity, total mass, etc. on the performance of the solar collector. Two experimental solar collectors with the parameters similar to those used in the simulations were built and they are going to be tested under the real climatic conditions. The acquired data will be used for fine tuning of the simulation models.

\section{Acknowledgements}

The research leading to the presented results was supported by the project OC10051 of Czech Ministry of Education and by the BUT project BD13102003 for young researchers. The co-author, the holder of Brno PhD Talent Financial Aid sponsored by Brno City Municipality, also gratefully acknowledges for that financial support.

\section{REFERENCES}

[1] Pinel P., Cruickshank C.A., Beausoleil-Morrison I., Wills A., A review of available methods for seasonal storage of solar thermal energy in residential applications, Renewable and Sustainable Energy Reviews 15 (2011) 3341- 3359.

[2] Jaber S., Ajib S, Optimum design of Trombe wall system in mediterranean region, Solar Energy 85, (2011)1891-1898.

[3] Zhao D.L., Li Y., Dai Y.J., Wang R.Z., Optimal study of a solar air heating system with pebble bed energy storage, Energy Conversion and Management 52 (2011) 2392-2400.

[4] Dutil Y., Rousse D.R., Ben Salah N., Lassue S., Zalewski L, A review on phasechange materials: Mathematical modeling and simulations. Renewable and Sustainable Energy Reviews 15, (2011) 112-130.

[5] Halawa E., Saman W., Thermal performance analysis of a phase change thermal storage unit for space heating. Renewable Energy 36, (2011) 259-264.

[6] Dolado P, Lazaro A, Marin J.M., Zalba B. Characterization of melting and solidification in a real-scale PCM-air heat exchanger: Experimental results and empirical model, Renewable Energy 36 (2011) 2906-2917.

[7] Alkilani M.M., Sopian K., Alghoul M.A., Sohif M., Ruslan M.H., Review of solar air collectors with thermal storage units, Renewable and Sustainable Energy Reviews 15 (2011) 1476-1490. 